\title{
Linking temperature to metabolism - the role of brown adipose tissue in humans
}

\author{
Jenny Backhaus, Leonie Rademacher, Alexander Iwen, Maren Waltl, Melanie Noll, \\ Hendrik Lehnert, Sebastian M. Schmid
}

\section{Background}

Brown adipose tissue (BAT) is a particular compartment of fat designated to produce heat through non-shivering thermogenesis (NST). Activated by moderate cold BAT metabolizes energy substrates like carbohydrates and fatty acids, thereby contributing to whole-body energy homeostasis. Against this background we studied the interaction between BAT activation and human energy homeostasis, focussing on glucose and fat metabolism. To further explore potential mechanisms linking BAT activity and metabolism, we evaluated endogenous stress systems activity as e.g. the sympathetic nervous system (SNS).

\section{Methods}

- 15 healthy normal-weight healthy men in a balanced cross-over within-person study design

- Two conditions: (i) thermoneutrality $\left(22^{\circ} \mathrm{C}\right)$; (ii) moderate cold $\left(18^{\circ} \mathrm{C}\right)$ following established BAT activation protocols

- Main outcome measures: Resting energy expenditure (indirect calorimetry), whole body insulin sensitivity and B-cell capacity

(by "Botnia clamp", i.e. combination of intravenous glucose tolerance test [ivGTT] followed by hyperinsulinaemic-euglycaemic clamp)
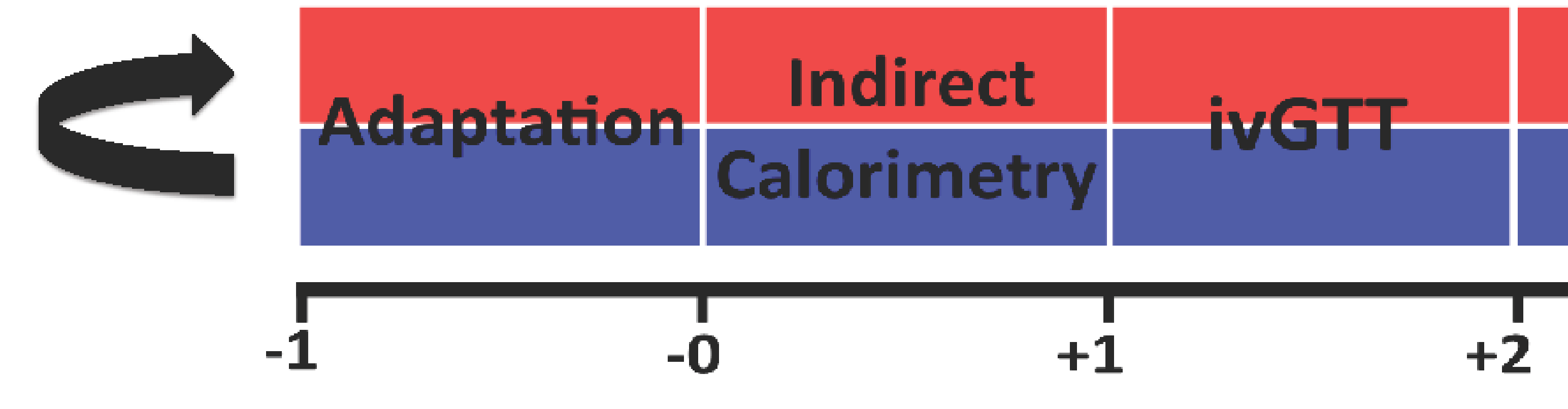

\begin{tabular}{|l|l|}
\hline Hyperinsulinaemic & Food \\
\hline Euglycaemic Clamp & Test \\
\hline+2 & +1
\end{tabular}
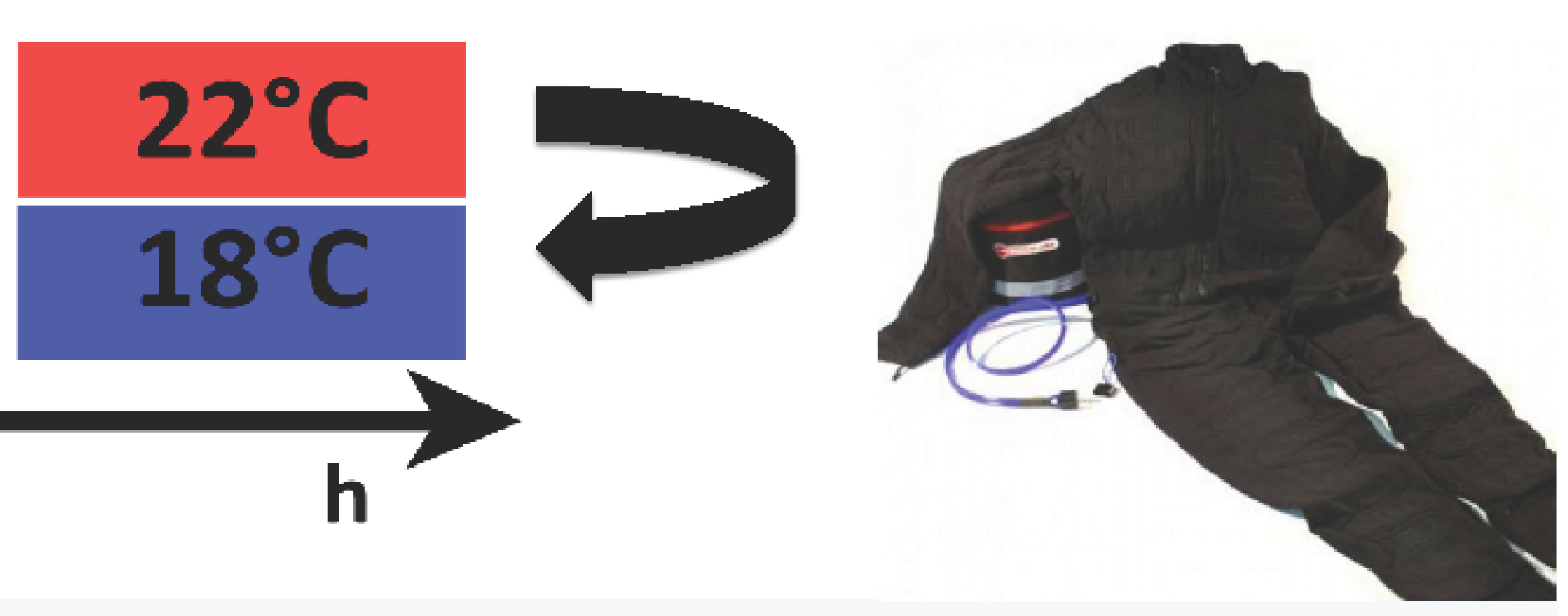

\section{Results}

PET-CT scans of a subset of 3 participants confirmed cold-induced activation of BAT. During cold-induced BAT activation insulin sensitivity as well as glucose utilisation increased by $\sim 20 \%$ as compared to thermoneutral conditions $(p<0.05)$. In contrast, pancreatic $\beta$-cell-capacity as indicated by First Phase Insulin Response (FPIR) revealed no significant differences between conditions. Acute BAT-activation was further accompanied by significantly higher triglyceride (TAG) levels $(p<.05)$. Norepinephrine and dopamine concentrations doubled from baseline during the BAT-activated state $(p<.0001)$, while epinephrine, HPA-axis activity, and pituitary-thyroid-axis activity were comparable between conditions.
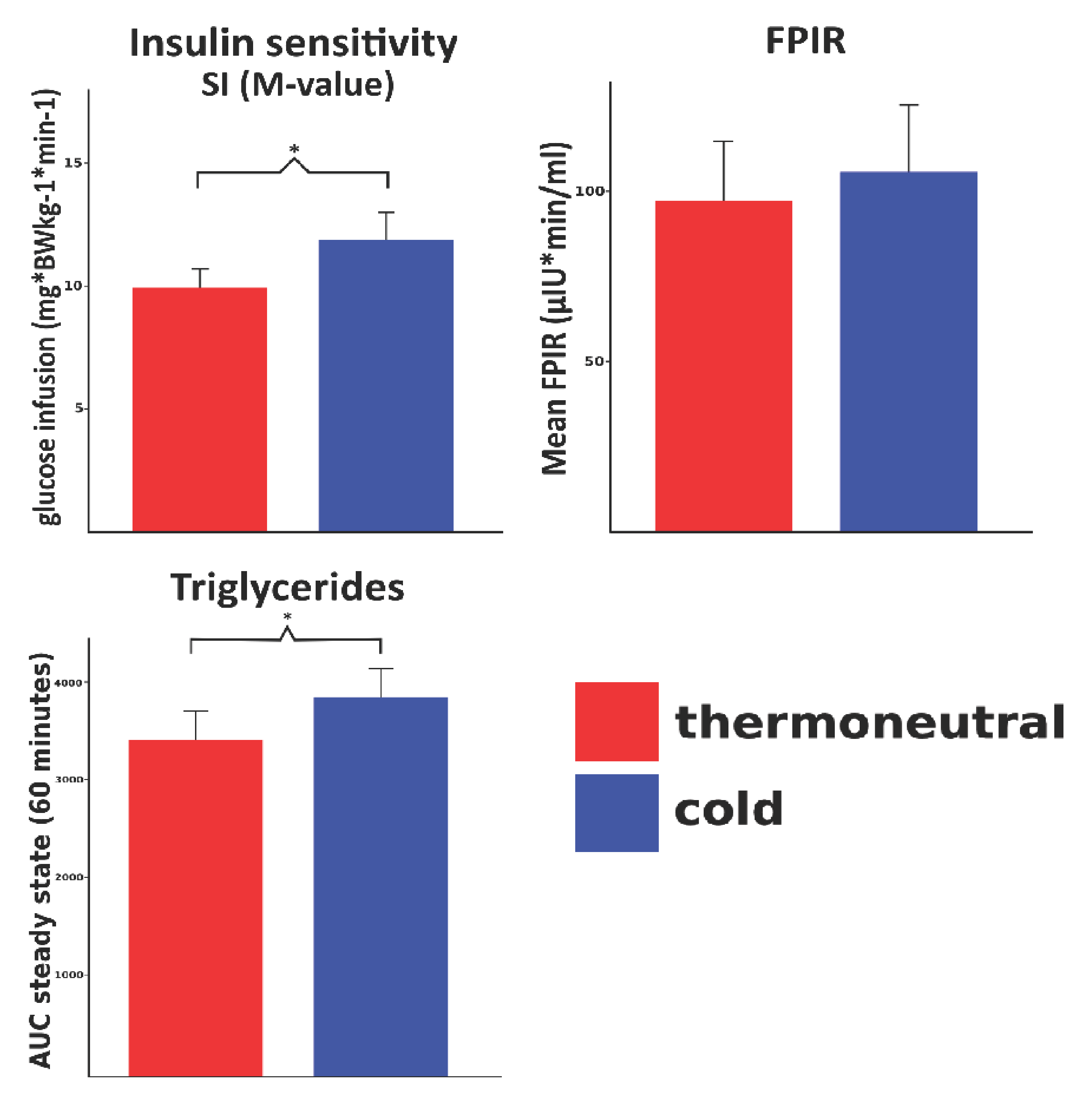
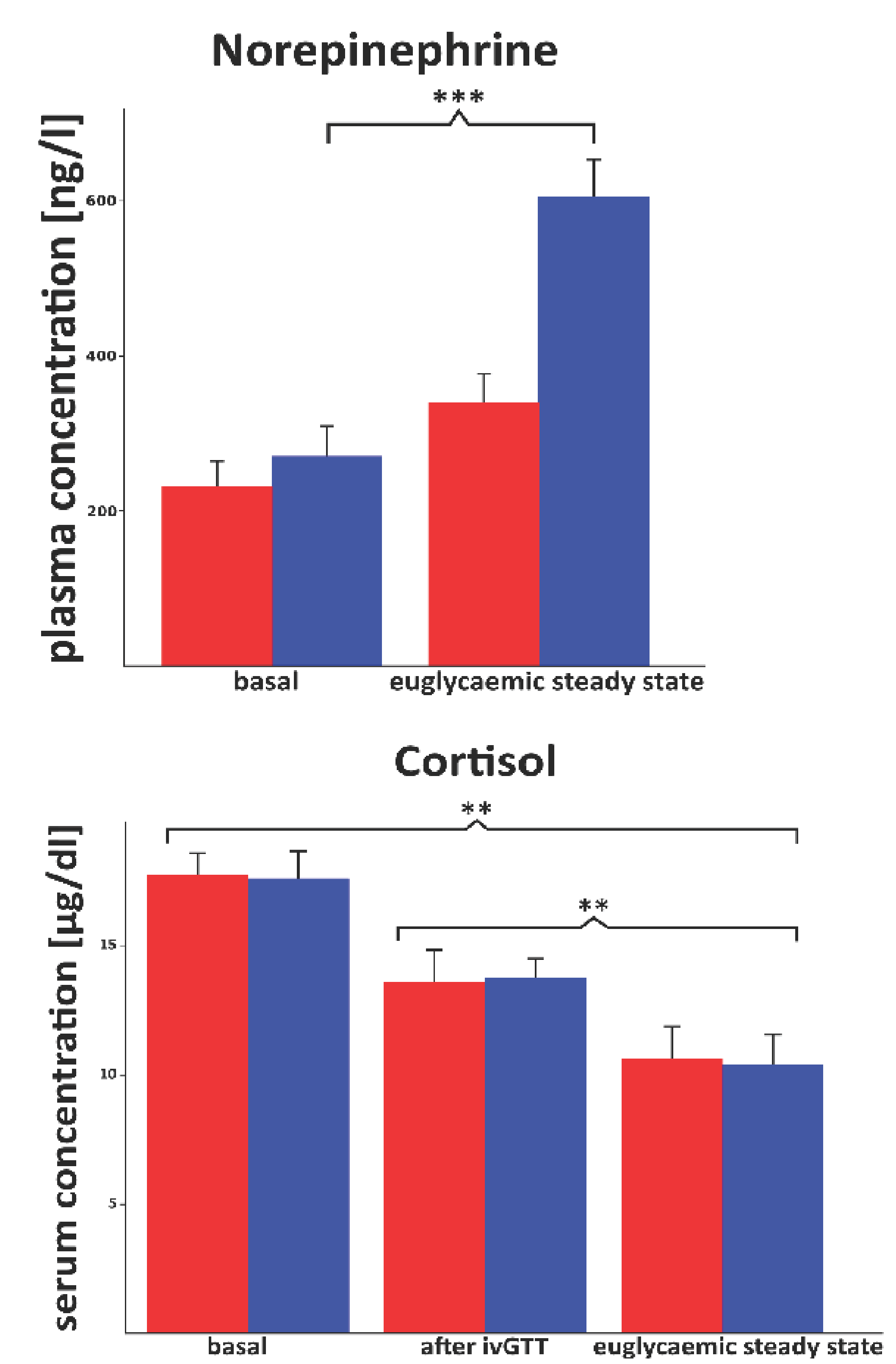

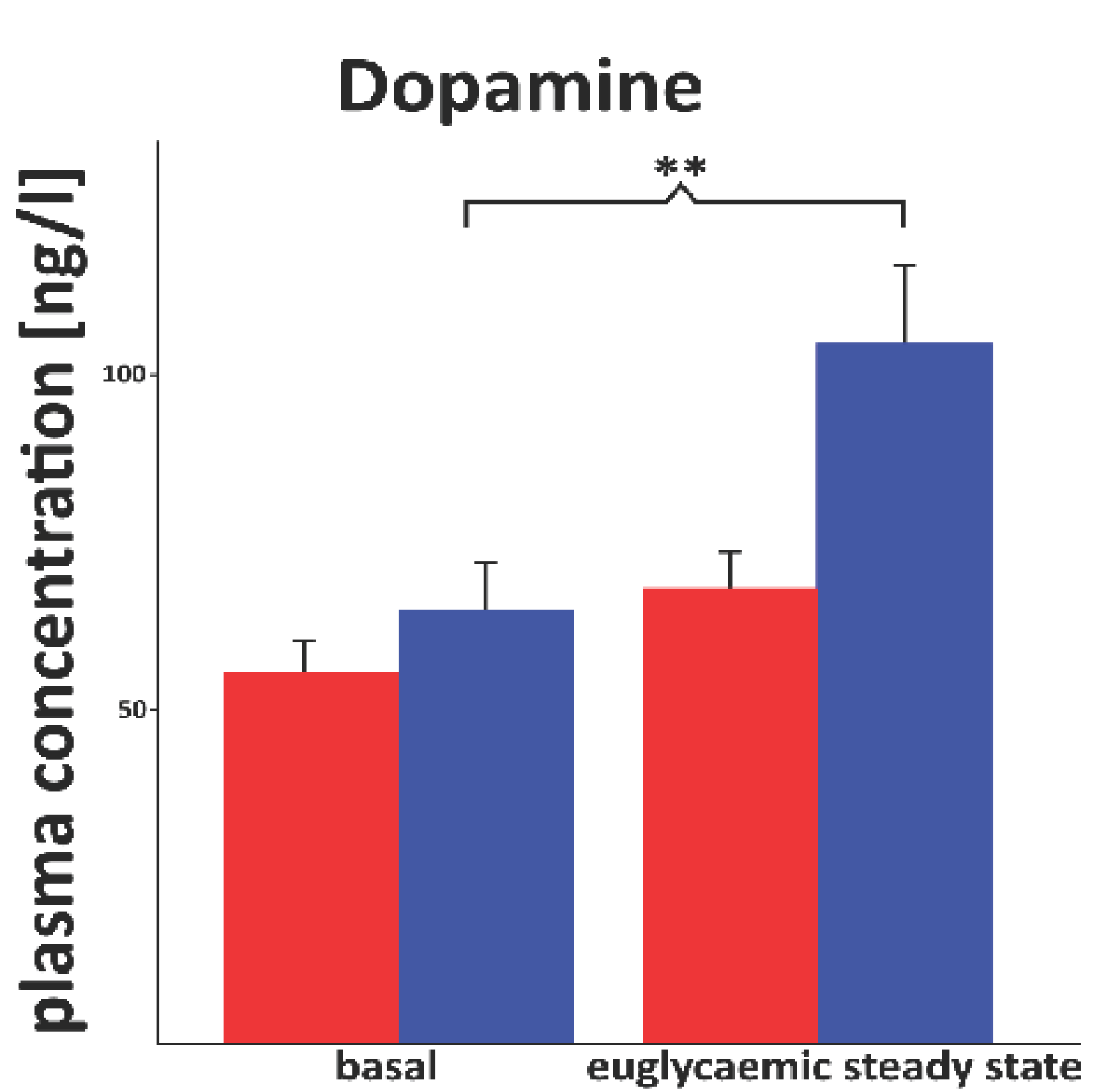

Epinephrine

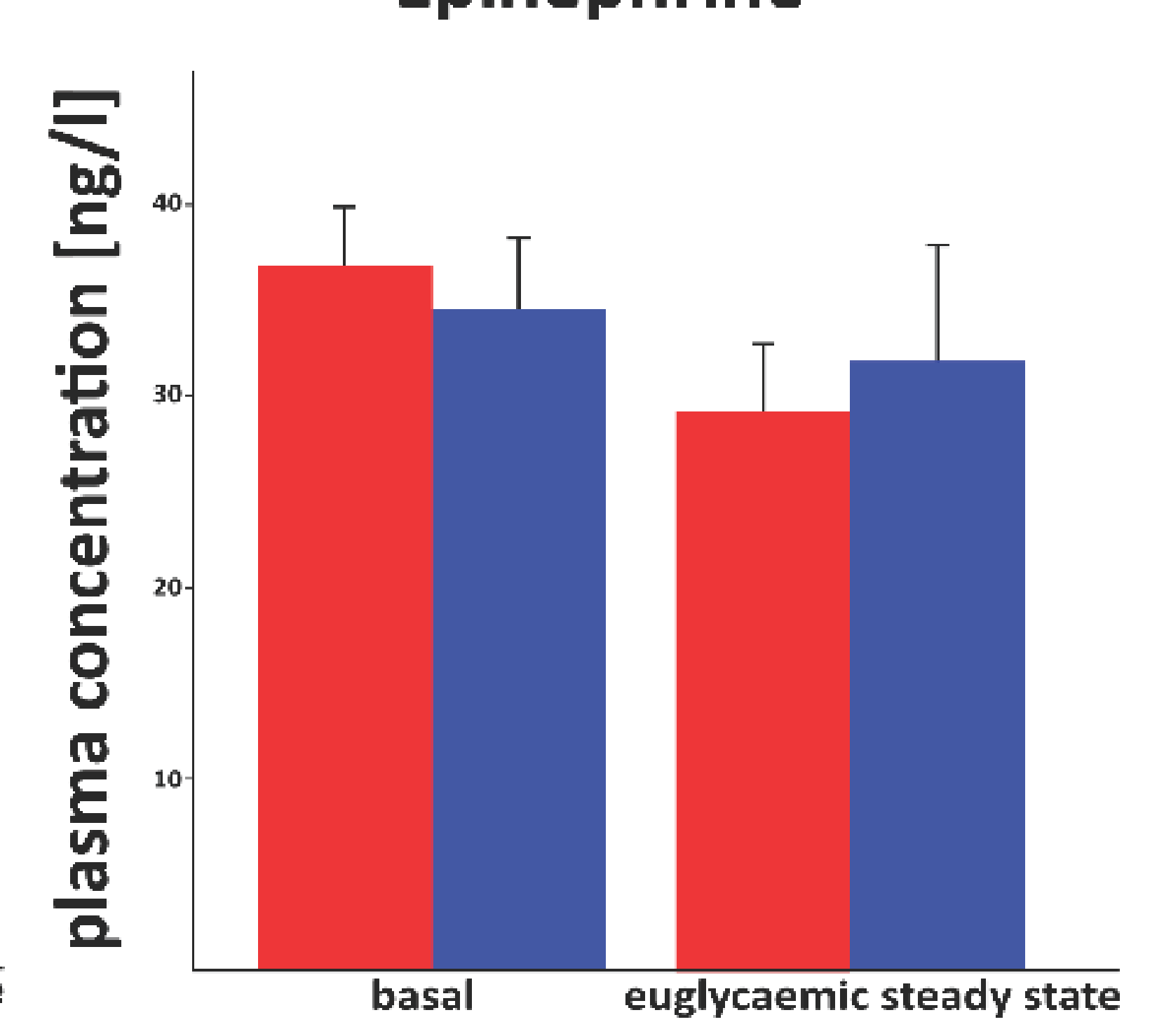

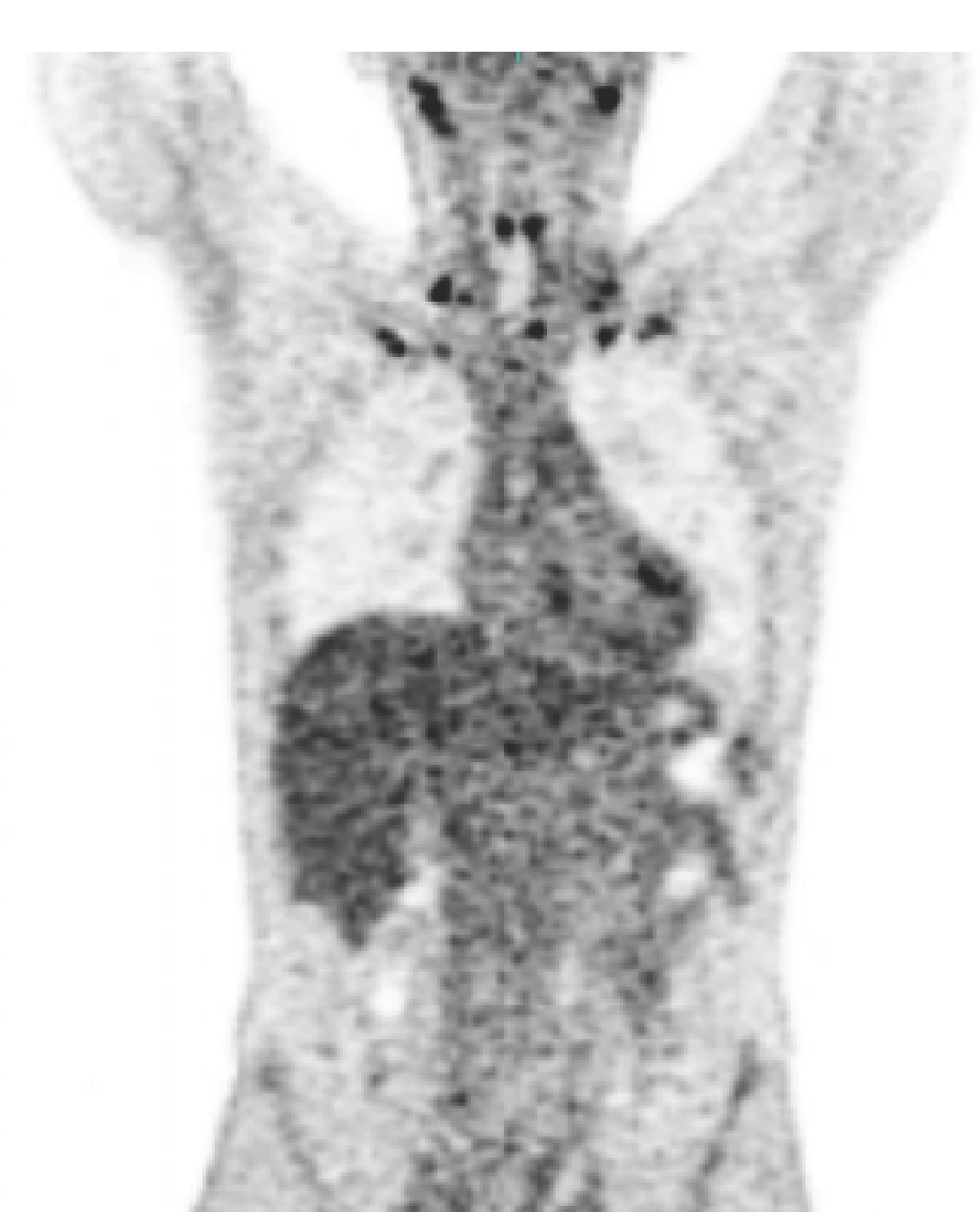

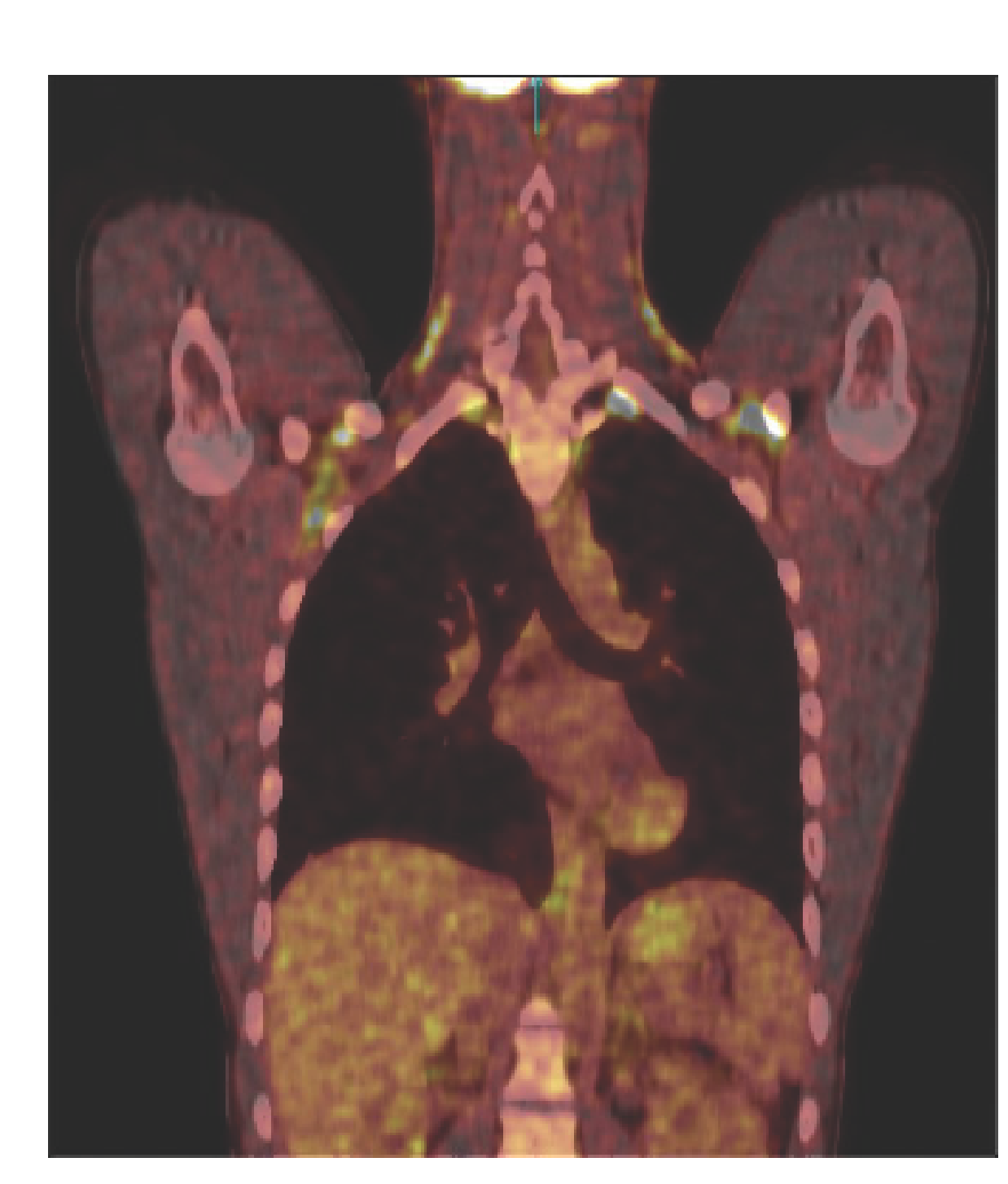

\section{Discussion}

Our findings of improved insulin sensitivity and glucose utilisation in normal weight men during cold-induced BAT activation clearly highlight its metabolic significance. Increased concentrations of TAGs during acute BAT activation might reflect an up-regulation of energy supply covering BAT's demand to maintain NST. Moreover, elevated plasma noradrenaline and dopamine levels suggest a distinct role of the SNS but not adrenergic or thyroidal stress response in mediating effects of BAT on human glucose metabolism. BAT activation might be a promising target for novel approaches in the treatment of metabolic diseases like obesity and Type 2 Diabetes. 